Recepción: 15 / 04 / 2017

Aceptación: 01 / 05 / 2017

Ciencias Médicas

Publicación: 15 / 05 / 2017

Caso Clínico

\title{
Exéresis de exostosis mandibular multilobular \\ bilateral: Reporte de Caso
}

\author{
Bilateral multilobular mandibular \\ exostoses: Case Report
}

\section{Excisão de exostosis multilobar mandibular \\ bilateral: Relato de Caso}

\author{
Peter W. Borbor-Silva \\ pietroxmilan@hotmail.com \\ Juan J. Macio-Pincay" \\ juan.maciop@ug.edu.ec \\ Nervo V. Medrano-Nuñez ${ }^{\text {"I }}$ \\ nervo.medranon@ug.edu.ec
}

Nestor A. Antepara-Lopez ${ }^{\mathrm{IV}}$ nestor.anteparal@ug.edu.ec

Correspondencia: juan.maciop@ug.edu.ec

Odontólogo; Universidad de Guayaquil, Ecuador.

Magister en Diseño Curricular; Diplomado en Docencia Superior; Doctor en Odontología; Universidad de Guayaquil, Ecuador.

III. Especialista en Implantologia Buco Maxilo Facial; Diplomado en Docencia Superior; Doctor en odontología; Universidad de Guayaquil, Ecuador.

Iv. Magister en Educación Superior; Especialista en Proyectos de Desarrollo Educativos y Sociales; Diplomado en Docencia Superior; Doctor en Odontología; Universidad de Guayaquil, Ecuador. 


\section{Resumen}

Las exostosis de los maxilares constituyen entidades benignas las cuales deben ser correctamente identificadas a través de un detallado diagnóstico diferencial. Son áreas vulnerables al trauma ya que pueden significar una zona de alto riesgo de osteonecrosis en pacientes que reciben bifosfonatos. Las exostosis son neoformaciones benignas localizadas o difusas del tejido óseo y para poder identificarlas es importante realizar un buen examen clínico y radiográfico, así como una completa anamnesis para establecer el diagnóstico y un eventual plan de tratamiento si el caso lo justifica, como veremos más adelante. El torus palatino podría ser el resultado de una leve isquemia crónica del periostio como resultado de una suave presión del septum nasal. Por otro lado, el torus mandibular sería consecuencia de la acción a la fuerza de torsión del arco de la mandíbula. Las exostosis óseas no requieren tratamiento a menos que sean de gran tamaño, compliquen las funciones de fonación, masticación, dicción, deglución o la posición normal de la lengua, generen desplazamiento dental o produzcan trauma y ulceración de la superficie mucosa, pero, especialmente, cuando imposibilitan la colocación y uso de las prótesis totales o removibles. Debemos establecer la exéresis de la exostosis mandibular multilobular bilateral en la clínica dentomaxilar de la Facultad Piloto de Odontología de la Universidad de Guayaquil. El resultado es favorable para el paciente con pronta recuperación. Cuando está indicado el tratamiento, la eliminación quirúrgica, mediante su tallado o remoción desde la base de la unión, es la técnica habitual recomendada para el paciente.

Palabras clave: Exostosis; periostio; multilobular. 
Peter W. Borbor-Silva; Juan J. Macio-Pincay; Nervo V. Medrano-Nuñez; Nestor A. Antepara-Lopez

\begin{abstract}
The maxillary exostosis are benign entities which must be properly identified through a detailed differential diagnosis. They are vulnerable to trauma areas because they can pose a high risk area of osteonecrosis in patients receiving bisphosphonates. The exostosis are benign neoplasms localized or diffuse bone tissue and for identification is important to make a good clinical and radiographic examination and a complete medical history to establish the diagnosis and eventual treatment plan if the case warrants, as discussed below. The palatal torus could be the result of mild chronic ischemia of the periosteum as a result of gentle pressure of the nasal septum. On the other hand, the mandibular torus would result from the action to the torque bow of the jaw. Bone exostosis not require treatment unless they are large, complicate functions of phonation, chewing, speech, swallowing or normal position of the tongue, generate dental displacement or produce trauma and ulceration of the mucosal surface, but especially when preclude placement and use of total or removable dentures. We must establish the excision of bilateral mandibular multilobar exostosis in the clinic dentomaxilar Pilot School of Dentistry at the University of Guayaquil. The result is favorable for the patient with early recovery. When treatment is indicated, surgical removal, by carving or removal from the base of the union, is the usual technique recommended for the patient.
\end{abstract}

Key words: Exostosis; periosteum; multilobular. 


\section{Resumo}

A exostose maxilar são entidades benignas que devem ser adequadamente identificadas através de um diagnóstico diferencial detalhado. Eles são vulneráveis a áreas de trauma porque podem representar uma área de alto risco de osteonecrose em pacientes que recebem bifosfonatos. A exostose é neoplasias benignas localizadas ou difusas tecido ósseo e de identificação é importante para fazer um bom exame clínico e radiográfico e uma história clínica completa para estabelecer o diagnóstico e plano de tratamento eventual, se o caso justifica, como discutido abaixo. O toro palatino pode ser o resultado de isquemia crônica leve do periósteo como resultado da pressão suave do septo nasal. Por outro lado, o toro mandibular resultaria da ação ao arco de torque da mandíbula. A exostose óssea não requer tratamento, a não ser que sejam grandes, compliquem funções de fonação, mastigação, fala, deglutição ou posição normal da língua, gerem deslocamento dental ou produzem trauma e ulceração da superfície mucosa, mas especialmente quando impedem a colocação eo uso de Próteses removíveis. Devemos estabelecer a excisão da exostose multilobar mandibular bilateral na clínica piloto dentomaxilar da Faculdade de Odontologia da Universidade de Guayaquil. O resultado é favorável para o paciente com recuperação precoce. Quando o tratamento é indicado, a remoção cirúrgica, por escultura ou remoção da base da união, é a técnica usual recomendada para o paciente.

Palavras chave: exostosis; periósteo; multilobar. 
Peter W. Borbor-Silva; Juan J. Macio-Pincay; Nervo V. Medrano-Nuñez; Nestor A. Antepara-Lopez

\section{Introducción.}

Desde el siglo pasado diferentes autores, como Fox (1809) y Danniels (1884), trataron de definir el Torus desde el punto de vista clínico e histopatológico, mientras que para los años 50 del presente siglo, Woo (1950) lo hace de acuerdo a su localización. (Lolymar, 2010)

La cavidad oral es el asentamiento de diversas patologías, que pueden alterar el estilo de vida. Entre esas patologías se encuentran los torus y otras exostosis. Son malformaciones con crecimiento excesivo del hueso y recubiertos por epitelio delgado sano. Se observan como un engrosamiento submucoso, duro y bien delimitados, recubierto por una mucosa pálida. Al corte, se muestra un tejido óseo denso con un patrón laminado, usualmente compuesto por hueso maduro, laminar, diseminado de osteocitos con espacios pequeños lleno de médula gruesa o estroma brovascular. Es mínima la actividad osteoblástica. (Arevalo, 2006)

Según Shafer y Levi en 1983 y Stafne en 1987, definen a los torus como protuberancias o exostósis que se encuentran en forma localizada en la región bucal, mientras que Antoniades y cols., y Sapp y cols., en 1998, coinciden en que los torus son excrecencias no neoplásicas, las cuales se pueden localizar en los maxilares provenientes del mismo hueso. (Lolymar, 2010)

El término torus, proviene del latín: eminencia o abultamiento. Fue utilizado primeramente por Kupfer y Besselhagen en1879, y se utiliza para designar las exostosis surgidas en la línea media del paladar y en la cara posterior e interna de la mandíbula. El torus palatino se presenta en la línea media del paladar duro y el torus mandibular en la cara lingual frente a bicúspides. A su vez las exostosis se encuentran a lo largo de la región vestibular del hueso alveolar. (Arevalo, 2006) 
A pesar que su etiología es desconocida, se han formulado diferentes teorías multifactoriales, pero no excluyentes, que explican la naturaleza genética (Suzuki y Sakai 1960; Sellevold, 1980; Seah,1995 ; Gorsky y cols, 1996; Nolte y cols,1997), ambiental (Pynn y cols, 1995; Seah , 1995; Nolte y cols, 1997), funcional (Johnson , 1959 ; Ossemberg ,1981 ; Pynn y cols , 1995; Czuszah y cols, 1996 ; Nolte y cols, 1997 ) en la formación de estas excrecencias óseas. (Marina, 2010)

La etiología es discutida, se han puntualizado factores genéticos, ambientales y la hiperfunción masticatoria Son de crecimiento lento a lo largo de la vida y por lo general asintomáticas y suelen iniciarse después dela pubertad. El tamaño y la forma pueden variar desde un guisante hasta una estructura multilobular de tamaño importante. Pueden apreciarse en una radiografía como un defecto radio opaco difuso. (Arevalo, 2006)

Pueden medir de tres a cuatro centímetros de diámetro. Los torus de mayor tamaño pueden entorpecer el movimiento de la lengua, interferir con el habla, limitar la colocación de aparatos protésicos y el mantenimiento de la higiene oral. Pueden producir ulceras que no cicatrizan y que pueden evolucionar a osteomielitis crónica. (Arevalo, 2006)

Garguilo y Arrocha en 1967 y Czuszah y cols., 1996, describen que después de realizar injertos gingivales libres, se podían observar la formación de torus o exostósis en áreas en las cuales se realizaron dichos injertos. Hegtvedt en 1990, propone tres teorías que explican la existencia de estas protuberancias óseas luego de realizar injertos libres de encía. (Adriana, 2010)

Los torus son excrecencias no neoplásicas del hueso cortical denso, comúnmente localizados en dos puntos específicos: línea media del paladar o región canino/premolar en la superficie lingual mandibular. (Rodríguez, 2014) 
Peter W. Borbor-Silva; Juan J. Macio-Pincay; Nervo V. Medrano-Nuñez; Nestor A. Antepara-Lopez

La etiología es discutida y se ha puntualizado factores genéticos, ambientales y la hiperfunción masticatoria como desencadenantes de su aparición. (Robles, 2014)

Los huesos maxilares no son ajenos a las patologías que se pueden presentar en el sistema esquelético. Algunas de esas condiciones y patologías son singulares por sus características clínicas, su distribución y prevalencia. (Castillo, 2008)

Los torus mandibulares se observan en la superficie lingual de la mandíbula en la zona de los premolares y las exostósis múltiples se observan en la superficie bucal del maxilar y de la mandíbula por debajo del pliegue mucobucal en la región molar. (Ruslan, 2009)

El torus como tumor óseo benigno puede localizarse en el maxilar a nivel del paladar, o en la mandíbula a nivel de las tablas internas; o puede aparecer en cualquier parte del esqueleto. (Castillo, 2008)

El TM es una exostosis o crecimiento óseo en la superficie lingual de la mandíbula. Este crecimiento ocurre generalmente cerca de la línea milohioidea, opuesto a los premolares, pero se puede extender del canino al primer molar. La mucosa que los recubre tiende a ser fina y no tolera por lo general las fuerzas de las prótesis que se colocan encima de ellos. (Castillo, 2008)

Los torus palatinos, los torus mandibulares (TM) y las exostosis de los maxilares son un claro ejemplo de ellos. Hasta la presente existen ideas especulativas acerca de su etiopatogenia, de los factores asociados, de su incidencia y prevalencia, de su necesidad de tratamiento, lo que puede crear confusión entre los clínicos tanto en diagnóstico como en el manejo. (Castillo, 2008)

Los torus son nódulos o protuberancias de diferentes formas configuradas por tejido óseo. Suelen crecer en la línea central del paladar (torus palatino) o la zona interior de la mandíbula (torus 
mandibular), concretamente donde se ubican los premolares, y lo habitual es que sean bilaterales. (Rosado, 2015)

Existen varios factores que pueden promover el desarrollo de los torus. Habitualmente se desarrollan al principio de la etapa adulta y están relacionados a bruxismo, masticación o factores genéticos. El tamaño de los torus varía con el tiempo y son de crecimiento lento. Suelen ser multilobulados y están recubiertos por una delgada capa de tejido oral la cual se lesiona con facilidad, especialmente por uso de prótesis confeccionadas sin detectar la presencia del torus. (Rosado, 2015)

Normalmente los torus no requieren ningún tipo de tratamiento, aunque puede ser necesaria su eliminación quirúrgica para evitar que interfieran en la realización de implantes dentales, la confección de prótesis removibles dento-muco soportadas para devolver la funcionalidad masticatoria al paciente, cuando son demasiado grandes, a tal punto que, comprometan la estética y llegan a provocar la inflamación de la mucosa o la ulceración de la misma, o si originan acumulación de alimentos a su alrededor, comprometiendo la higiene bucal y ocasionando halitosis. (Rosado, 2015)

Son de crecimiento lento, a lo largo de la vida. Radiográficamente se observa como un defecto radiopaco, difuso, que puede medir de 3 a 4 cm de diámetro. (Frías, 2014)

Los torus son un tipo de exóstosis óseas (crecimiento anormal de hueso, no tumoral, que produce una protuberancia sobre la superficie), que pueden aparecer tanto en la cara interna de la mandíbula como en el paladar. Tienen un crecimiento muy lento, y hasta en un $90 \%$ de los casos pueden ser bilaterales. Histológicamente muestran la estructura del hueso cortical maduro. Radiológicamente aparecen como lesiones radio-opacas difusas. (Polanco, 2012) 
Peter W. Borbor-Silva; Juan J. Macio-Pincay; Nervo V. Medrano-Nuñez; Nestor A. Antepara-Lopez

La incidencia del torus de la mandíbula es baja en el 6\% a $12.5 \%$ entre caucásicos y en los habitantes de la llanura africana. De manera contraria, algunos autores reportan una prevalencia mucho más elevada en la Costa Atlántica Colombiana. (Marquez, 2008)

Aparecen en la línea media del paladar duro, con formas diversas (planos, fusiformes, nodulares o polilobulados). La mucosa que los cubre suele estar íntegra, a veces algo más pálida, aunque si se traumatiza se ulcera con cierta facilidad y puede tardar en cicatrizar. (Polanco, 2012)

Son frecuentes, aparecen hasta en un $20 \%$ de la población. El tamaño puede variar desde tan pequeños que pasan inadvertidos y se detectan por palpación, hasta otros que llegan a ocupar todo el paladar interfiriendo con el habla normal. Habitualmente se localizan en la porción central de la línea media del paladar, aunque también pueden aparecer en la parte anterior o posterior. (Polanco, 2012)

Los de mayor tamaño pueden entorpecer el movimiento de la lengua, interferir en el habla y limitar la colocación de aparatos protésicos. Es recomendable en caso de producirse úlceras concomitantes en el torus y no asentar bien la prótesis removible por ese defecto; la excisión quirúrgica del mismo. (Rodríguez, 2014)

Los torus son exóstosis óseas benignas redondeadas, con superficie lisa y formada por hueso nodular denso. Su localización habitual es en la línea media del paladar o en las superficies linguales de la mandíbula; esta localización nos permite diferenciarlos de los osteomas. (Rodríguez, 2014)

Son lesiones relativamente frecuentes que llegan a aparecer hasta en el 25\% de la población, en el caso de los palatinos y en un $10 \%$ en los mandibulares. Son asintomáticas y suelen ser un hallazgo causal en el momento de la colocación de una prótesis. Sólo se tratan quirúrgicamente cuando lo exige un tratamiento protético. (Rodríguez, 2014) 
También pueden llevar a otras lesiones, como hiperplasia e incluso llegara ser factor etiológico de enfermedad bucal maligna. Susuki y Sakai en 1960 al estudiar los aspectos familiares de los torus, encontraron que cuando ambos padres tenían torus palatino o torus mandibulares, la prevalencia en sus hijos eran 63\% y 58\%, respectivamente. (Arevalo, 2006)

El fibroma osicante o cementante maduro que ha producido un abultamiento del paladar cortical puede imitar una exostosis. Sin embargo, en ese caso las radiografías periapicales muestran un reborde radiotransparente fino que rodea la lesión fibroósea madura. También pueden imitar un torus o una exostosis, el hematoma subperióstico osificado, el callo óseo no resuelto, el osteoma, el osteosarcoma precoz y el condrosarcoma precoz. (Avendaño, 2006)

Los torus y las exostosis se pueden asociar a ciertas enfermedades, como la adenomatosis del colon, el síndrome de Mafucci, la enfermedad de Olier y los osteocondromas múltiples. (Avendaño, 2006)

Al igual que la exóstosis y osteomas, los torus son considerados como lesiones tumorales benignas, aunque no son neoplasias verdaderas. En realidad son excrecencias no neoplásicas de hueso cortical denso, comúnmente localizado en dos puntos específicos: línea media del paladar o región canino/ premolar en la superficie lingual mandibular. (Robles, 2014)

Estas malformaciones de hueso están recubiertas por epitelio delgado sano. Se observan como un engrosamiento submucoso, duro y bien delimitados, recubierto por una mucosa pálida. Al corte se observa un tejido óseo denso con un patrón laminado, usualmente compuesto por hueso maduro, laminar, diseminado por osteocitos con espacios pequeños llenos de médula gruesa o estroma fibrovascular. (Frías, 2014) 
Peter W. Borbor-Silva; Juan J. Macio-Pincay; Nervo V. Medrano-Nuñez; Nestor A. Antepara-Lopez

El torus palatino se presenta en la línea media del paladar duro y el torus mandibular en la cara lingual frente a bicúspides. (Robles, 2014)

La etiología es discutida y se ha puntualizado factores genéticos, ambientales y la hiperfunción masticatoria. Son de crecimiento lento a lo largo de la vida y por lo general asintomáticas y suele iniciarse después de la pubertad. (Frías, 2014)

El tamaño y forma de los torus puede variar desde un guisante hasta una estructura multilobular de tamaño importante. Puede apreciarse en una radiografía como un defecto radiopaco difuso y puede medir de 3 ó 4 cm de diámetro. (Rodríguez, 2014)

El torus de mayor tamaño puede entorpecer el movimiento de la lengua, interferir en el habla, limitar la colocación de aparatos protésicos y el mantenimiento de higiene oral. Puede producir úlceras que no cicatrizan y que pueden evolucionar a osteomielitis crónica. (Robles, 2014)

El fibroma osificante o cementoma madura que ha producido un abultamiento del paladar cortical puede imitar una exóstosis. Sin embargo, en ese caso las radiografías periapicales muestran un reborde radiotransparente fino que rodea la lesión fibroósea madura. (Frías, 2014)

También puede imitar un torus o una exóstosis, el hematoma subperióstico osificado, el callo óseo no resuelto, el osteoma, el osteosarcoma precoz y el condrosarcoma precoz. (Rodríguez, 2014)

Los torus y exostósis normalmente aparecen en mujeres de la tercera década de vida, edad, en una proporción de mujer: hombre de 2:1 (Shafer y Levi 1987). En niños son sumamente raros. (Pynn, 1995) 
El presente reporte de caso tiene como objetivo establecer la excéresis de la exostosis mandibular multilobular bilateral en la clínica dentomaxilar de la Facultad Piloto de Odontología de la Universidad de Guayaquil.

\section{Reporte de Caso.}

\section{Motivo de consulta:}

El paciente llegó a la facultad recomendado por la Dra. Rocío Centeno y nos manifiesta que desea mejorar su salud oral por lo que nos dice: "Quiero arreglarme los dientes”. Se le realizaron tratamientos quirúrgicos entre ellos: extracciones dentales, tratamientos periodontales y por último la exéresis de exostosis mandibular con el fin de su rehabilitación bucal.

\section{Anamnesis:}

El paciente presenta hipertensión y está bajo medicación, tomando warfarina y cardio aspirina, no presenta ninguna otra enfermedad sistemática o infectocontagiosa, no padece alergias a ningún medicamento. Además, el paciente indica que dejó de fumar y tomar hace más de 20 años. En antecedentes patológicos familiares el paciente no refiere ningún antecedente. En sus signos vitales la presión se encuentra en 130-80, la frecuencia cardiaca se encuentra en 80 lat. x min. 36.5 ${ }^{\circ} \mathrm{C}$ y la frecuencia respiratoria es de 18 respiraciones por minuto. Al examen extra oral no presenta ninguna patología aparente y al examinar los tejidos blandos no se encuentra ninguna anomalía.

\section{Descripción del Odontograma:}


Descripción: Ausencia de piezas 18, 17, 16, 15, 13, 12, 11, 21, 22, 24, 25, 26, 27, 28, 38, 37, 36, 35, 34, ,46, 47 ;Caries en la cara mesial de pieza 14: Caries en cara oclusal de pieza 48; Caries en cara vestibular de pieza 44.

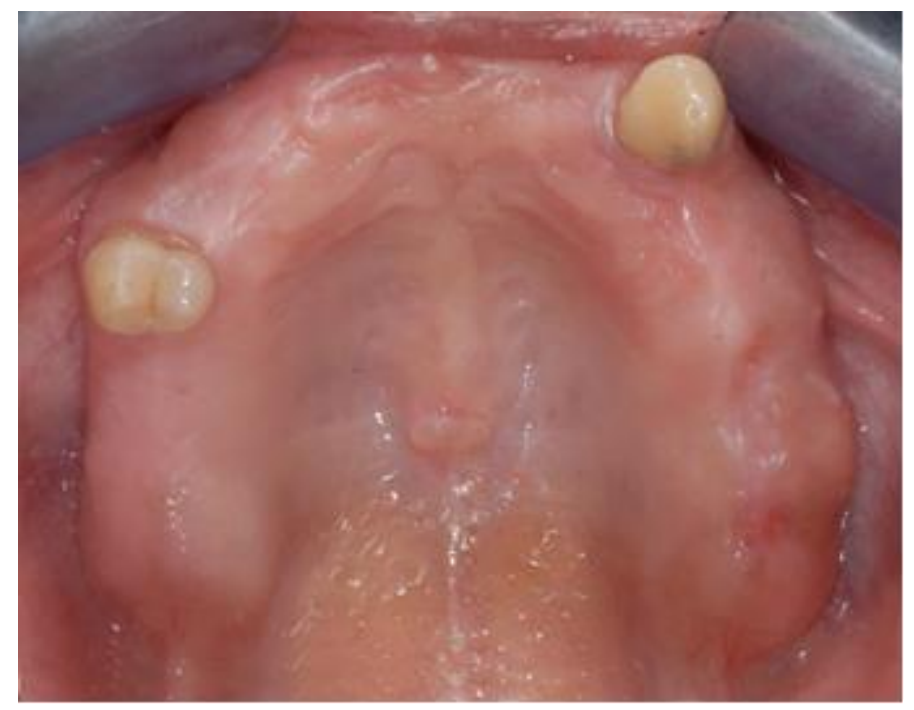

Figura $N^{\circ}$ 1.- Fotos intraorales; Análisis: Se observa la presencia de pieza 14 y 2

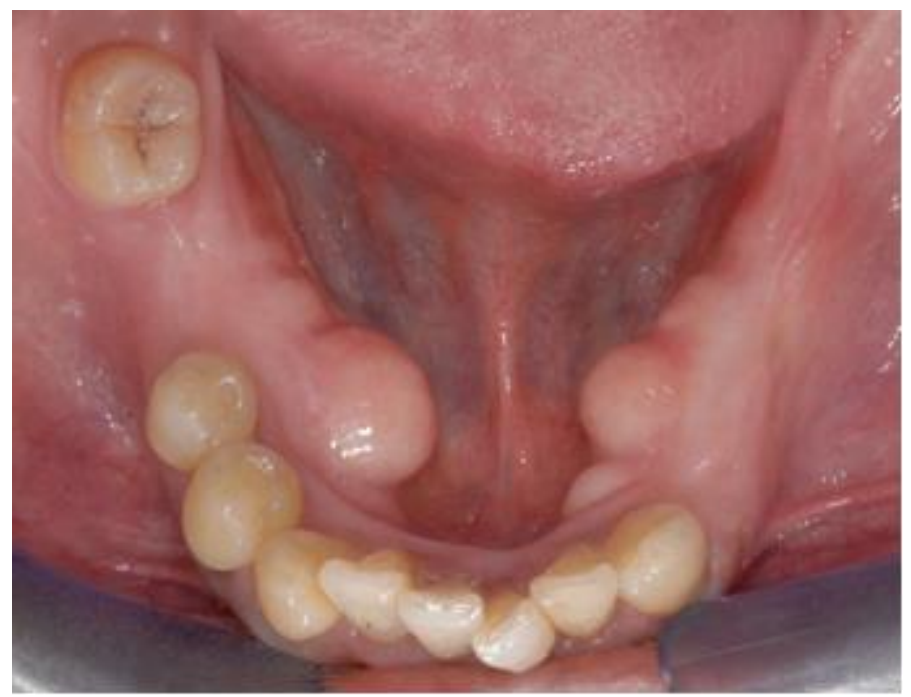

Figura $N^{\circ}$ 2.- Foto de arcada inferior; Análisis: Se observa la presencia de piezas 48, 45, 44, 43, 42, 41, 31, 32, 33. Presencia de exostosis mandibular bilateral multilobular. 


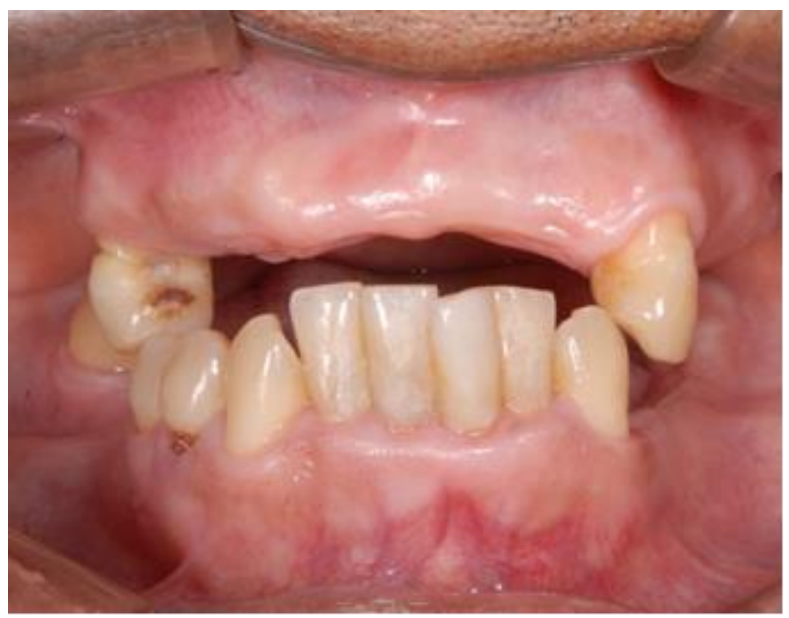

Figura $N^{\circ}$ 3.- Imagen frontal ambas arcadas en oclusión.; Análisis: Se observa caries mesial en pieza 14. Por ausencia de piezas superiores se observa extrusión de piezas inferiores.

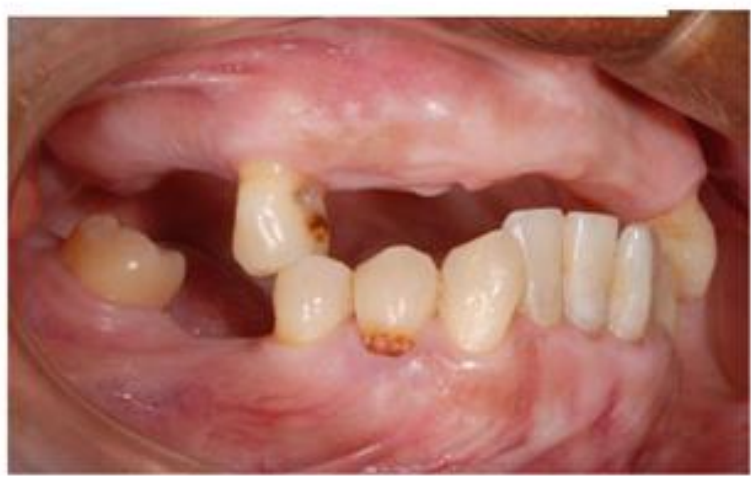

Figura $N^{\circ}$ 4.- Imagen lateral derecha; Análisis: No se observa clase de Angle por ausencia de piezas dentarias.

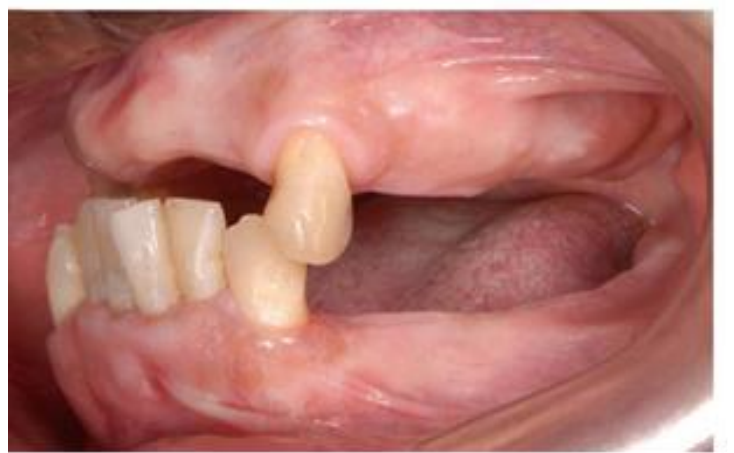

Figura $N^{\circ}$ 5.- Imagen lateral izquierda; Análisis: No se observa clase de Angle por ausencia de piezas dentarias. 
Peter W. Borbor-Silva; Juan J. Macio-Pincay; Nervo V. Medrano-Nuñez; Nestor A. Antepara-Lopez

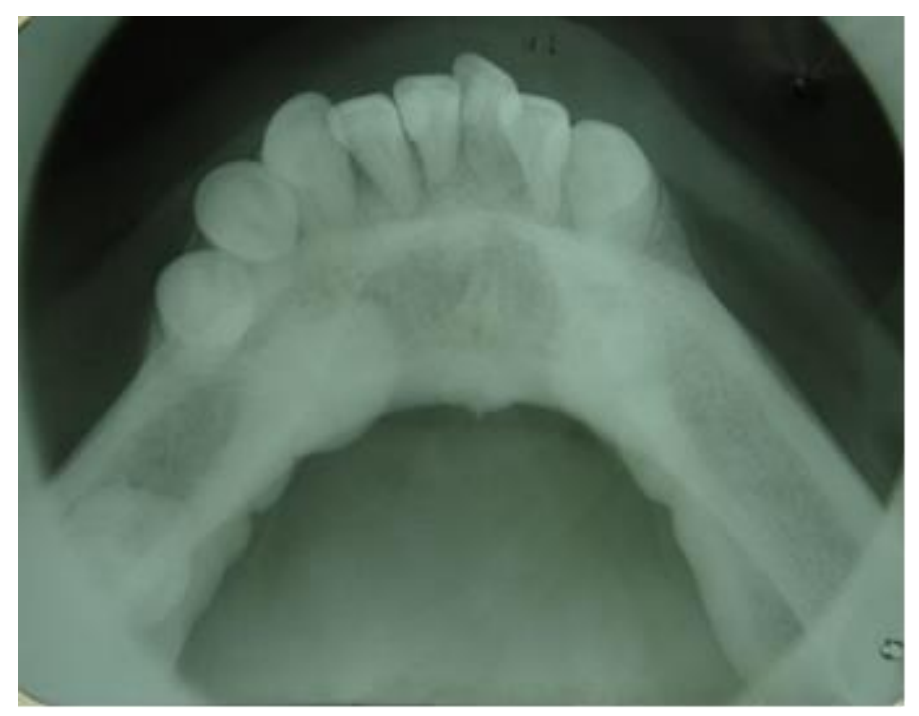

Figura $N^{\circ}$ 6.- Rx oclusal del paciente; Análisis: Sombras radiopacas a nivel del cuerpo de la mandíbula compatible con exostosis mandibular.

\section{Diagnóstico.}

Exéresis de exostosis mandibular bilateral multilobular.

\section{Pronóstico}

Favorable para el paciente.

\section{Planes de tratamiento}

a. Excéresis de exostosis mandibular con escoplo y martillo.

b. Excéresis de exostosis mandibular con pieza de mano.

c. No se realiza la excéresis de la exostosis y se realiza una prótesis dental con rebase. 


\section{Tratamiento}

La cirugía de extirpación de torus no es complicada, aunque la recuperación puede ser lenta. El odontólogo normalmente utilizará un anestésico local y luego reducirá o eliminará el torus mandibular con instrumentos especiales. (Marin, 2015)

Después de quitar los torus, el médico sutura la zona. Los pacientes deben esperar por lo menos una semana para su recuperación inicial, momento en el que tienen que volver para una postevaluación. La recuperación total tomará tres a cuatro semanas, aunque un paciente será capaz de realizar la mayoría de sus actividades habituales durante el período de recuperación. (Marin, 2015)

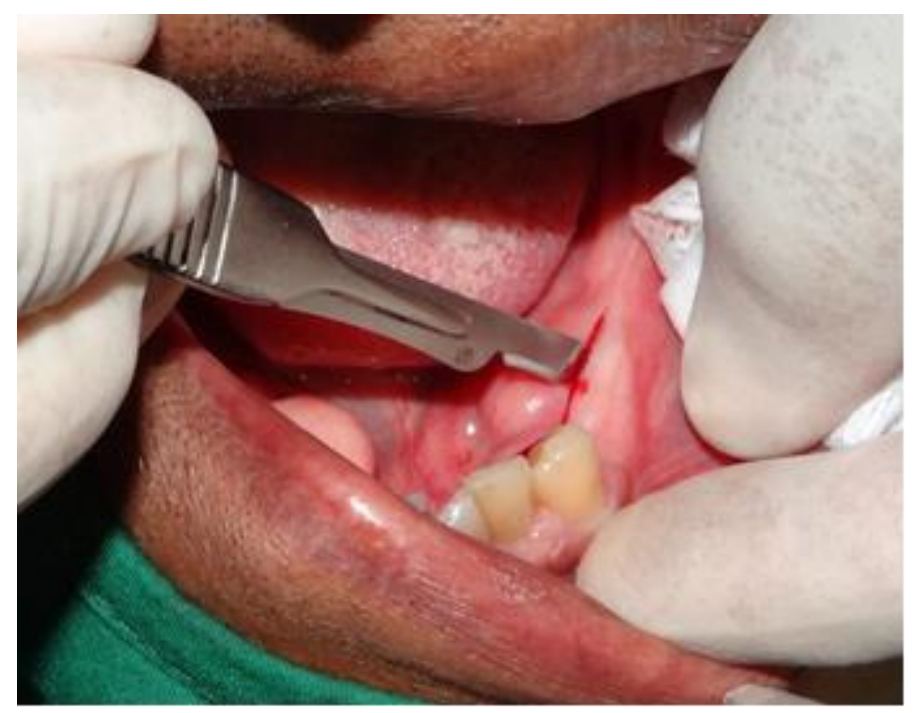

Figura $N^{\circ}$ 7.- Incisión con bisturí hoja \# 15 
Peter W. Borbor-Silva; Juan J. Macio-Pincay; Nervo V. Medrano-Nuñez; Nestor A. Antepara-Lopez

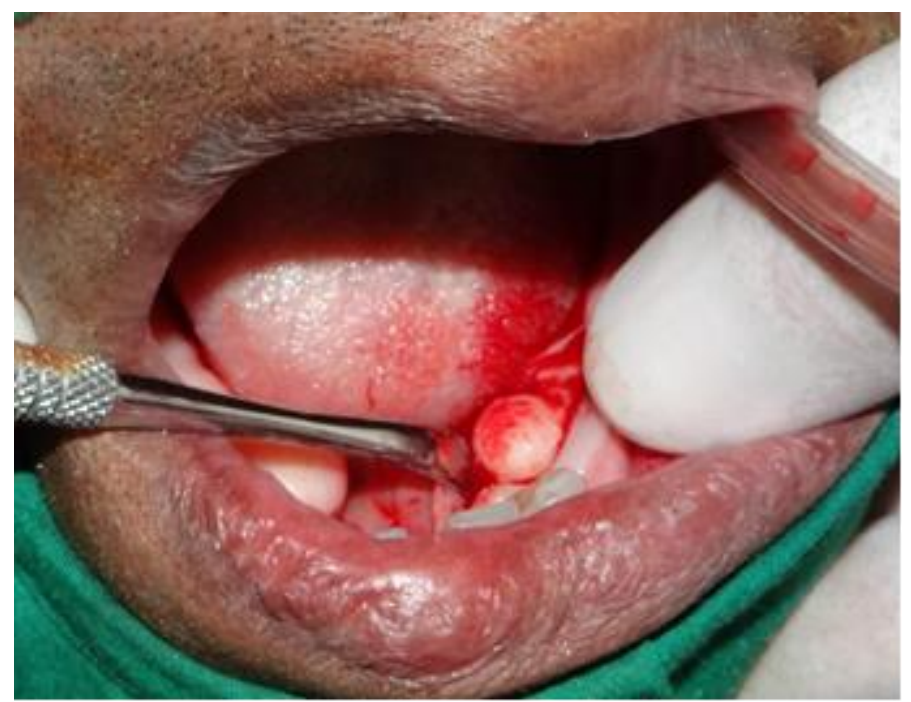

Figura $N^{\circ}$ 8.- Sindesmotomía y debridación; Análisis: Separación de la mucosa, debridación, levantamiento de colgajo y exposición de la exostosis mandibular

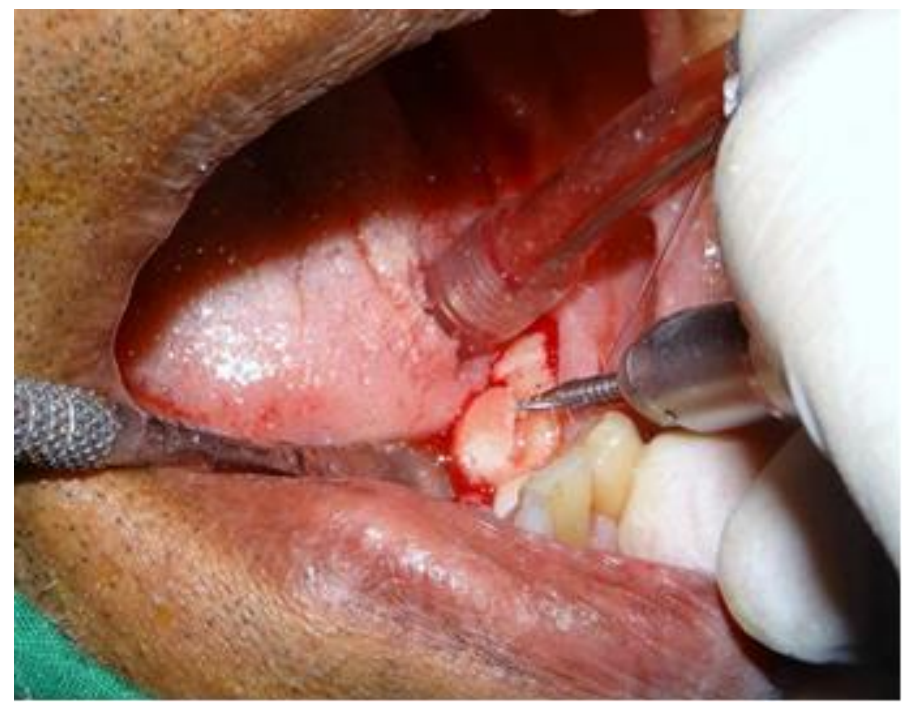

Figura $N^{\circ}$ 9.- Osteotomía; Análisis: Exéresis de la exostosis mandibular por medio de osteotomía 


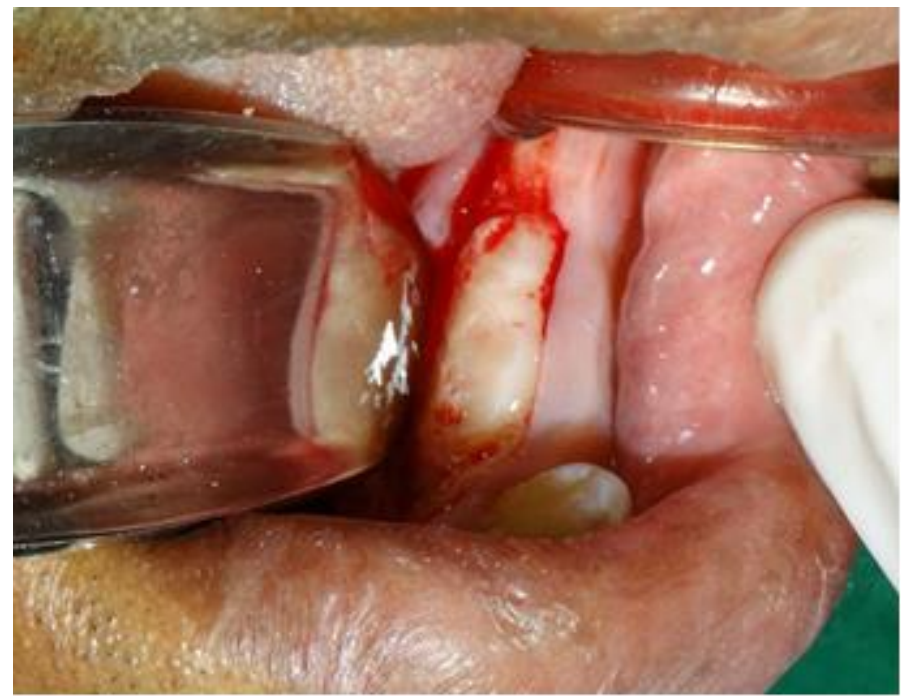

Figura $N^{\circ}$ 10.- Osteotomía finalizada; Análisis: Osteotomía terminada

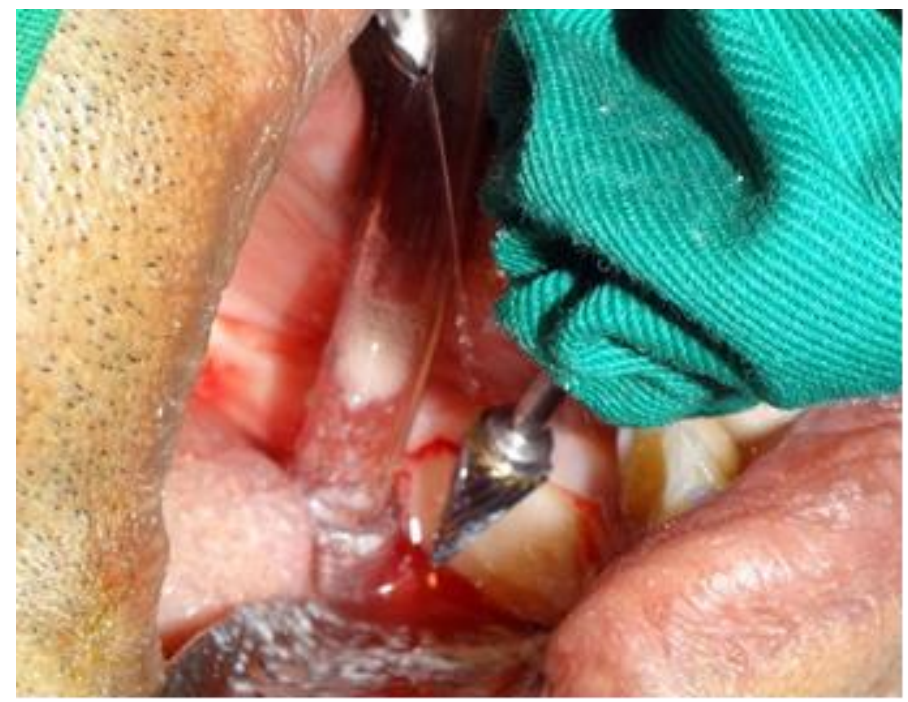

Figura $N^{\circ}$ 10.- Regularización de hueso; Análisis: Alisado de hueso 
Peter W. Borbor-Silva; Juan J. Macio-Pincay; Nervo V. Medrano-Nuñez; Nestor A. Antepara-Lopez

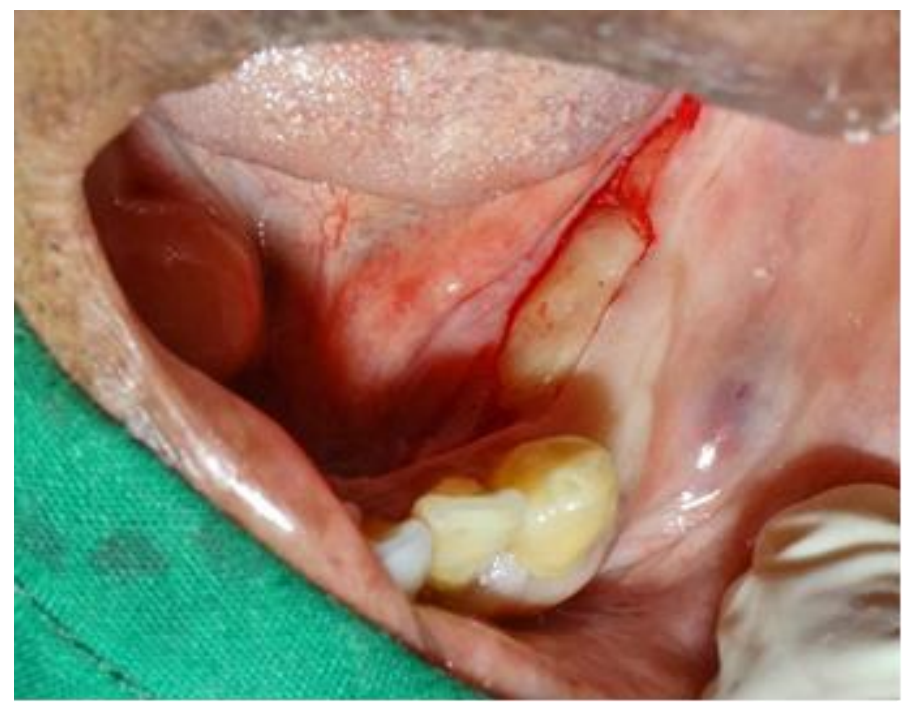

Figura $N^{\circ}$ 11.- Alisamiento de hueso finalizado; Análisis: Regularización de hueso terminado

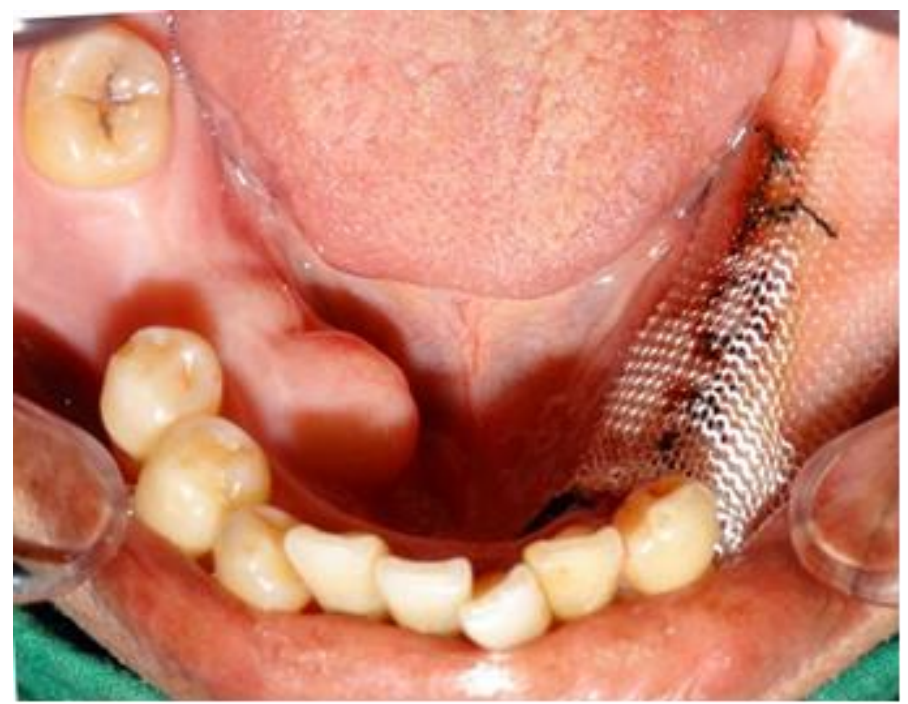

Figura $N^{\circ}$ 12.- Aplicación de surgicel; Análisis: Colocación de membrana de surgicel 


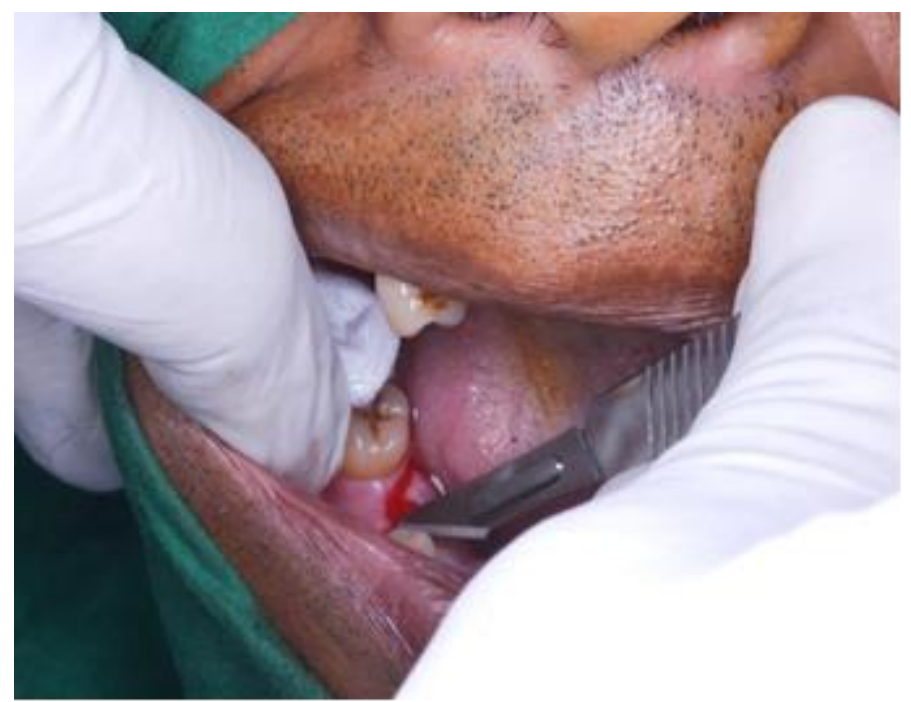

Figura $N^{\circ}$ 13.- Incisión lado derecho; Análisis: Incisión en el área quirúrgica con hoja de bisturí \#15

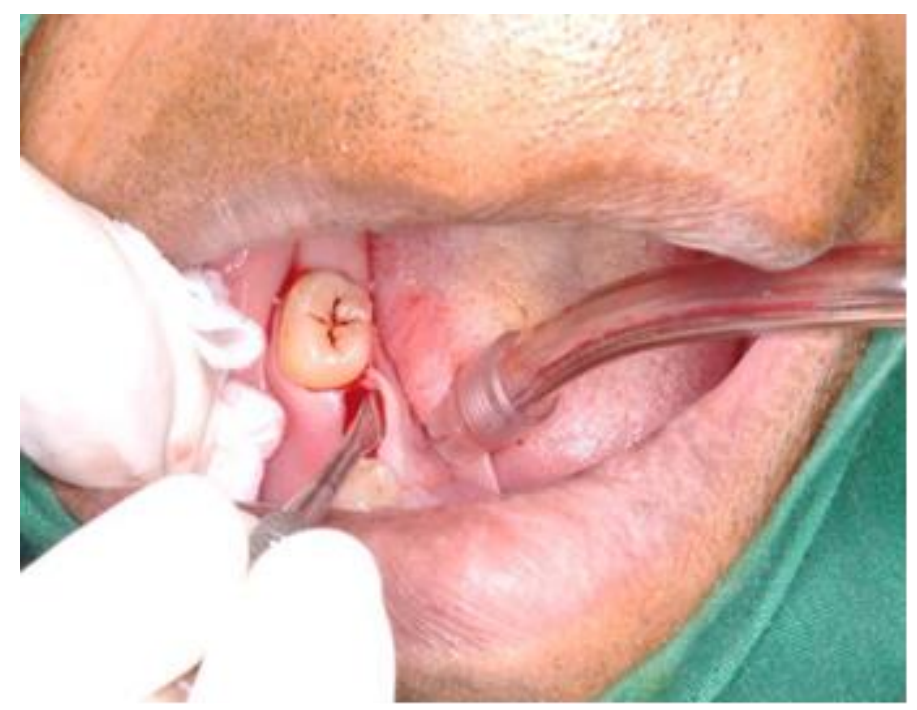

Figura $N^{\circ}$ 14.- Sindesmotomía lado derecho; Análisis: Debridación de mucosa y levantamiento de colgajo 
Peter W. Borbor-Silva; Juan J. Macio-Pincay; Nervo V. Medrano-Nuñez; Nestor A. Antepara-Lopez

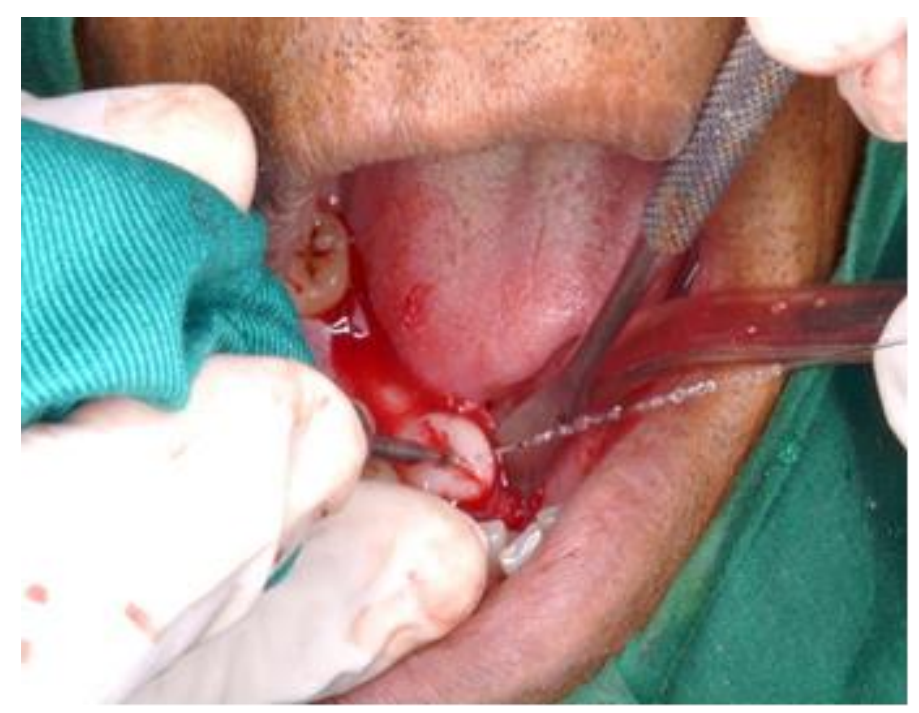

Figura $N^{\circ}$ 15.- Osteotomía lado derecho; Análisis: Exéresis de exostosis mandibular lado derecho

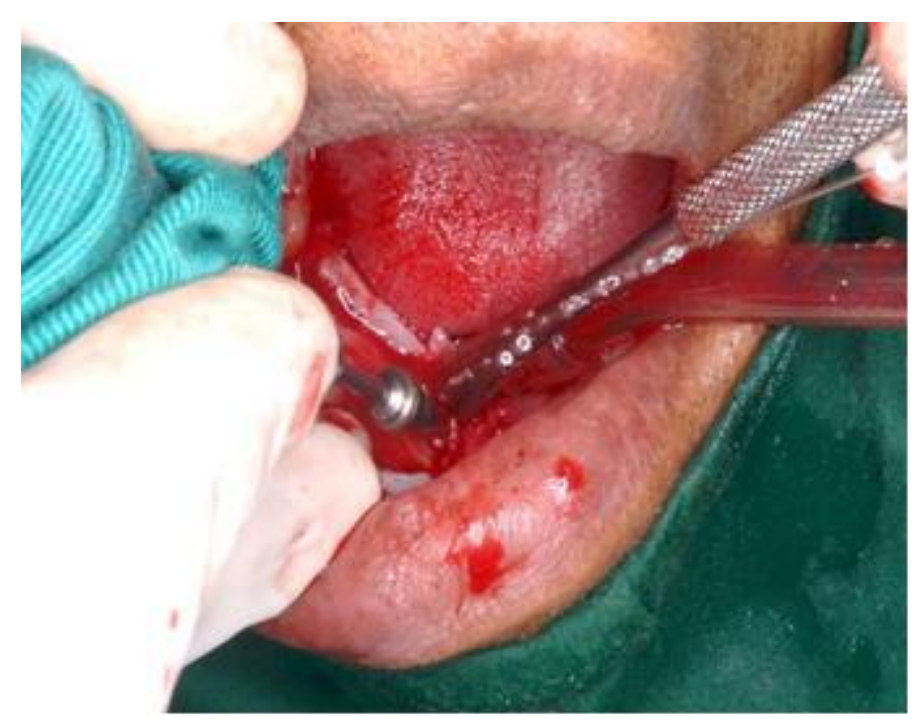

Figura $N^{\circ}$ 16.- Regularización de hueso lado derecho; Análisis: Regularización de hueso 


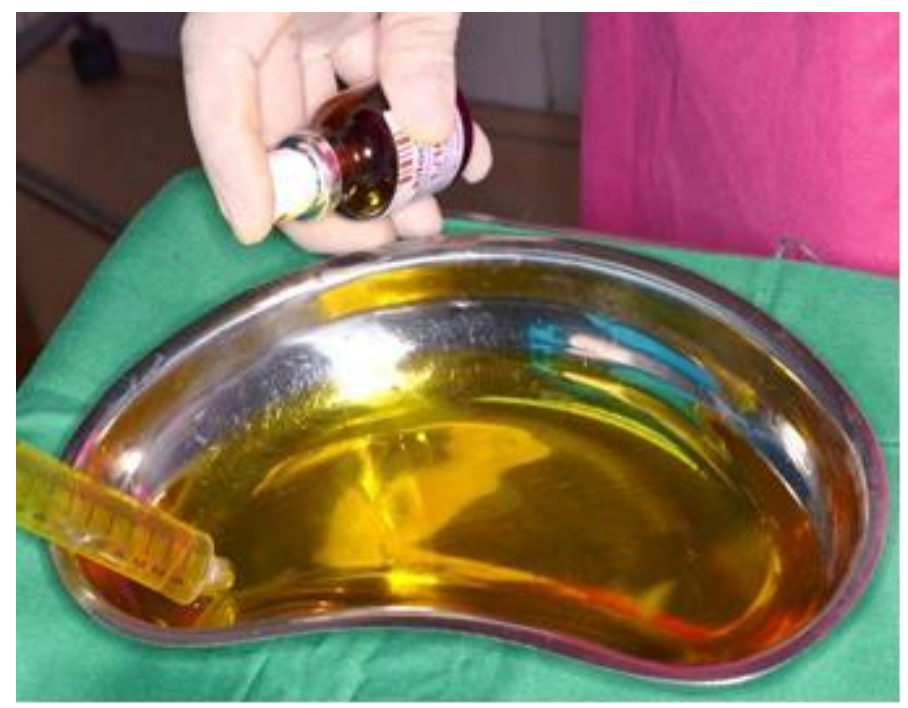

Figura $N^{\circ}$ 16.- Aplicación de rifosina al suero fisiológico; Análisis: Aplicación de rifosina en suero fisiológico

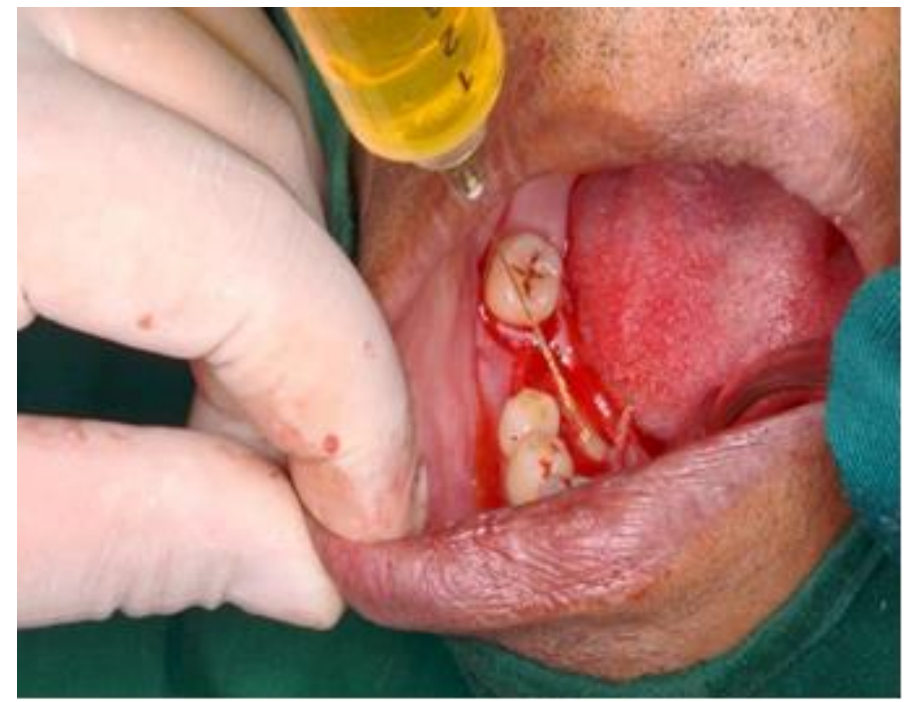

Figura $N^{\circ}$ 17.- Irrigación de la zona operatoria; Análisis: Irrigación con rifosina+ suero fisiológico en la zona quirúrgica 
Peter W. Borbor-Silva; Juan J. Macio-Pincay; Nervo V. Medrano-Nuñez; Nestor A. Antepara-Lopez

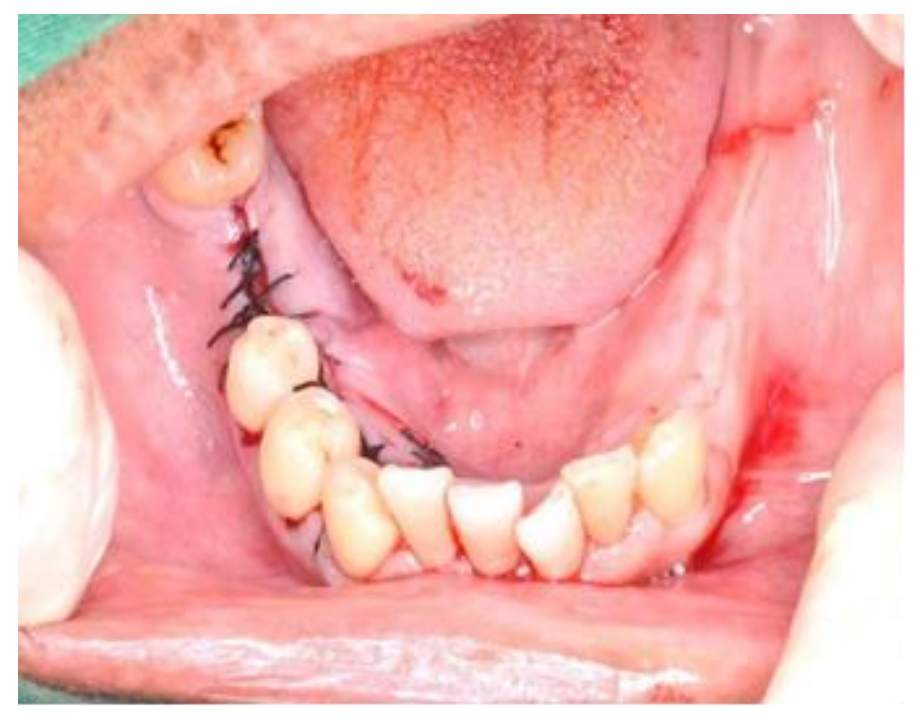

Figura $N^{\circ}$ 18.- Sutura finalizada; Análisis: Sutura finalizada, punto individual

\section{Discusión.}

Diferentes tipos de exóstosis se han descrito, siendo los torus palatino y el torus mandibular, las dos más comunes exóstosis intraorales; las cuales frecuentemente son visibles en las radiografías periapicales dentales. (Rodríguez, 2014)

Diversos estudios corroboran el predominio del torus palatino, frente al torus mandibular. No obstante, otros autores como Bruce y Al Quaran encontraron una mayor prevalencia de torus mandibular con respecto al torus palatino. No hay explicaciones para esta discrepancia de prevalencia. (Frías, 2014)

Suele detectarse entre personas de 20 a 30 años, disminuyendo en los mayores de 50 años. Diversos autores opinan que los sujetos con torus mandibulares, exhibían una mayor prevalencia de dientes desgastados y disfunción temporomandibular, que se ha relacionado con el bruxismo. (Robles, 2014) 
Por lo regular no se realiza tratamiento al Torus, aunque en ocasiones puede ser de tal tamaño y forma que es difícil elaborar una prótesis parcial o total. Se puede intentar construir la prótesis sobre la prominencia ósea, siempre y cuando no sea muy grande la lesión. Si la lesión es grande, la aparatología puede ser imposible y se hace necesario un tratamiento quirúrgico. Otras indicaciones para escisión quirúrgica pueden ser: razones psicológicas, trauma continuado, interferencia con el habla y la masticación.

\section{Conclusiones.}

Los torus o exostósis constituyen excrecencias óseas no neoplásicas las cuales tienen una etiología desconocida, los cuales pueden diagnósticarse a través del examen clínico, radiográfico e histopatológico. A pesar de que estas excrecencias óseas no tienen significado patológico como tal se han propuesto diversas indicaciones para su tratamiento con la finalidad de satisfacer todas las necesidades del paciente. Es de gran importancia estar familiarizado con éstas estructuras óseas ya que tienden a confundirse muchas veces con lesiones neoplásicas benignas y malignas y está en nuestro campo conocerlas desde todo punto de vista para así poder planificar el plan de tratamiento de nuestros pacientes.

\section{Bibliografía.}

Antoniades Dz \& cols.:1998 Concurrence of torus palatinus with palatal and bucal exostoses: case and review of the literature. Oral Surg Oral Med Oral Pathol, 85: 552-557.

Sapp Philips \& cols.:1998 Patología Oral y Maxilofacial Contemporánea. Madrid.

Shafer W.G. \& Levi B.M: 1986 Tratado de Patología Bucal .Cuarta edición, México: Editorial Interamericana.

Stafne: 1987. Diagnóstico Radiológico en Odontología. Quinta edición, Buenos Aires, Editorial Panamericana.

Figun M. \& Garino R.: 1988 Anatomía Odontológica Funcional y Aplicada. Tercera ediciòn,Argentina. Editorial El Ateneo. 
Peter W. Borbor-Silva; Juan J. Macio-Pincay; Nervo V. Medrano-Nuñez; Nestor A. Antepara-Lopez

Seah Y.H.: 1995.Torus palatinus and torus mandibularis: a review of the literature. Australian Dental Journal 40(5):318-321.

Suzuki M. \&Sakai T.: 1960. A familial study of the torus palatinus and torus mandibularis.Am J Phys Anthropol.18:263-272.

Sellevold B.J.:1980 Mandibular torus morphology. Am J Phys Anthropol. 53:569.

Gorsky M. \& cols.: 1996 Prevalence of torus palatinus in a popolution of young and adults Israelits.Arch Oral Biol,41: 623-625.

Nolte A. \& Schirren C.G: 1997 Torus Mandibularis.Hautarzt. 48: 414-416.

Pynn B. \& cols: 1995. Tori Mandibularis: a case report and review of the literature. Journal of the Canadian Dental Association 61-12:1057-1066.

Johnson Otis: 1950 The tori and the Masticatori stress. J Pros Den 9-6:975-977.

Ossemberg, N.S: 1981. Mandibular torus: a synthesis of new previously reported data and discussion of its cause. Am J Phys Anthropol 1-52.

Czuszak Ch \& cols: 1996. Development of an exostosis following a free gingival graft: case reports. Journal of Periodontology. 67:3 250-253.

Garguilo A. \& Arrocha R.: 1967. Histochemical evaluation of free gingival grafts. Periodontics, 5:285-291.

Hegtvedt A. \& cols.: 1990. Skin grafts vestibuloplasty exostósis. Oral Surg Oral Med Oral Pathol. 69:149152.

Schelegel: 1996.Patologìa Médico Quirúrgica bucofacial. Tercera edición Madrid.

Eggen S. \& Natving B: 1991.Variation in torus mandibularis prevalence in Norway. Community Dent Oral Epidemiol. 19: 32-35.

Miller Ernest: 1975. Prótesis parcial removible. Primera edición, México, Editorial Interamericana.

Henderson D. \& Steefel V. 1988: Prótesis Parcial Removible según Mc Craken. Sexta edición, Argentina, Editorial Mundi.

Volchansky: 1996 Clinical study of mandibular exostoses in South Africa Caucasoids. Journal of Dental Association o South Africa. 41: 139-141. 